\title{
CHARACTERIZATION OF COMMUTATIVE ALGEBRAS EMBEDDED INTO THE ALGEBRA OF SMOOTH OPERATORS
}

\author{
TOMASZ CIAŚ
}

\begin{abstract}
The paper deal with the noncommutative Fréchet ${ }^{*}$-algebra $\mathcal{L}\left(s^{\prime}, s\right)$ of the so-called smooth opertors, i.e. linear and continuous operators acting from the space $s^{\prime}$ of slowly increasing sequences to the Fréchet space $s$ of rapidly decreasing sequences. By a canonical identification, this algebra of smooth operators can be also seen as the algebra of the rapidly decreasing matrices. We give a full description of closed commutative ${ }^{*}$-subalgebras of this algebra and we show that every closed subspace of $s$ with basis is isomorphic (as a Fréchet space) to some closed commutative *-subalgebra of $\mathcal{L}\left(s^{\prime}, s\right)$. As a consequence, we give some equivalent formulation of the long-standing Quasi-equivalence Conjecture for closed subspaces of $s$.
\end{abstract}

\section{INTRODUCTION}

In this paper we consider some specific noncommutative Fréchet algebra with involution - known, for istance, as the algebra $\mathcal{L}\left(s^{\prime}, s\right)$ of smooth operators or as the algebra

$$
\mathcal{K}_{\infty}:=\left\{\left(x_{j, k}\right)_{j, k \in \mathbb{N}} \in \mathbb{C}^{\mathbb{N}^{2}}: \sup _{j, k \in \mathbb{N}}\left|x_{j, k}\right| j^{q} k^{q}<\infty \quad \text { for all } q \in \mathbb{N}_{0}\right\}
$$

of rapidly decreasing matrices. Our main goal is to solve the following problems:

(A) characterize Fréchet ${ }^{*}$-algebras isomorphic to closed commutative ${ }^{*}$-subalgebras of $\mathcal{L}\left(s^{\prime}, s\right)$ (Theorem 4);

(B) characterize the underlying Féchet spaces for the class of closed commutative ${ }^{*}$-subalgebras of $\mathcal{L}\left(s^{\prime}, s\right)$ (Theorem 7).

By definition, $\mathcal{L}\left(s^{\prime}, s\right)$ is the Fréchet *-algebra consisting of all linear and continuous operators from the space

$$
s^{\prime}:=\left\{\left(\xi_{j}\right)_{j \in \mathbb{N}} \in \mathbb{C}^{\mathbb{N}}: \sup _{j \in \mathbb{N}}\left|\xi_{j}\right| j^{-q}<\infty \quad \text { for some } q \in \mathbb{N}_{0}\right\}
$$

of slowly increasing sequences (this an inductive limit of Banach spaces) to the Fréchet space

$$
s:=\left\{\left(\xi_{j}\right)_{j \in \mathbb{N}} \in \mathbb{C}^{\mathbb{N}}: \sup _{j \in \mathbb{N}}\left|\xi_{j}\right| j^{q}<\infty \text { for all } q \in \mathbb{N}_{0}\right\}
$$

of rapidly decreasing sequences. Since there is a natural inclusion $\mathcal{L}\left(s^{\prime}, s\right)$ into $\mathcal{B}\left(\ell_{2}\right)$, the space $\mathcal{L}\left(s^{\prime}, s\right)$ is indeed a ${ }^{*}$-algebra with composition of operators and hilbertain involution taken from $\mathcal{B}\left(\ell_{2}\right)$. Futhermore, $\mathcal{L}\left(s^{\prime}, s\right)$ with its natural topology of the uniform convergence on bounded subsets of $s^{\prime}$ is a Fréchet space.

We prove, in particular, that the class of closed commutative ${ }^{*}$-subalgebras of $\mathcal{L}\left(s^{\prime}, s\right)$ is - in the sense of an isomorphism of Fréchet *-algebras - exactly the class of Köthe sequence algebras which are closed under taking square roots (Definition 3) and at the same time isomorphic (as Fréchet spaces) to closed subspaces of $s$ (Theorem 4). This can be easily expressed by conditions on the corresponding Köthe matrices. Moreover, from the solution of Problem (A) we get the solution of Problem (B): the

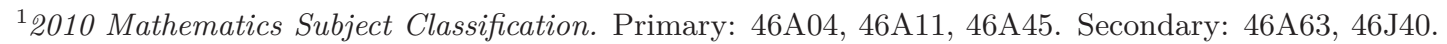

Key words and phrases: Köthe sequence algebras, nuclear Fréchet spaces, property (DN), structure of Fréchet algebras, smooth operators.

The research of the author was supported by the National Center of Science, grant no. 2013/09/N/ST1/04410. 
underlying Fréchet spaces for closed commutative ${ }^{*}$-subalgebras of $\mathcal{L}\left(s^{\prime}, s\right)$ are just closed subspaces of $s$ with basis (Theorem 17).

It has been already shown in [2, Th. 4.8] that every infinite-dimensional closed commutative *subalgebra $X$ of $\mathcal{L}\left(s^{\prime}, s\right)$ is isomorphic as a Fréchet *-algebra to a Köthe sequence algebra

$$
\lambda^{\infty}\left(\left\|P_{j}\right\|_{q}\right):=\left\{\left(\xi_{j}\right)_{j \in \mathbb{N}} \in \mathbb{C}^{\mathbb{N}}: \sup _{j \in \mathbb{N}}\left|\xi_{j}\right|\left\|P_{j}\right\|_{q}<\infty \text { for all } q \in \mathbb{N}_{0}\right\}
$$

where $\left(P_{j}\right)_{j \in \mathbb{N}}$ is a suitable sequence of pairwise orthogonal, nonzero, self-adjoint projections belonging to $X$ and $\left(\|\cdot\|_{q}\right)_{q \in \mathbb{N}_{0}}$ is a sequence of continuous norms on $\mathcal{L}\left(s^{\prime}, s\right)$ which determines the Fréchet space topology of $\mathcal{L}\left(s^{\prime}, s\right)$. Hence, in order to solve problems (A) and (B), we needed to "understand" matrices $\left(\left\|P_{j}\right\|_{q}\right)_{j \in \mathbb{N}, q \in \mathbb{N}_{0}}$, where $\left(P_{j}\right)_{j \in \mathbb{N}}$ are pairwise orthogonal, nonzero, self-adjoint projections belonging to $\mathcal{L}\left(s^{\prime}, s\right)$. The key step in solutions of our problems was to show that for every nuclear Fréchet space with the so-called dominating Hilbert norm $\|\cdot\|$ there is a topological embedding $V: X \hookrightarrow s$ such that $\|V x\|_{\ell_{2}}=\|x\|$ for every $x \in X$ (Theorem 14).

In this context, it is worth mentioning that, by [4, Th. 6.2], a Köthe sequence algebra of the form (31) is isomorphic to some closed *-subalgebra of the algebra $s$ if and only if it is isomorphic as a Fréchet space to a complemented subspace of $s$. One would think that every closed commutative *-subalgebra of $\mathcal{L}\left(s^{\prime}, s\right)$ is of this type. Theorem [4, Th. 6.9] shows that this is not true (in the proof we give a concrete contrexample) and the present paper shows that indeed - taking into account the representation (3) - the class of the underlying Fréchet spaces for closed commutative *-subalgebras of $\mathcal{L}\left(s^{\prime}, s\right)$ is as big as possible, i.e. as we mentioned above, it consists of all closed subspaces of $s$ with basis.

The algebra of smooth operators is a quite natural object. It can be seen as a kind of a noncommutative analogon of the commutative Fréchet algebra $s$ (look at the definition of $\mathcal{K}_{\infty}$ above) and, in particular, it is isomorphic as a Fréchet space to $s$ (see [12, Lemma 31.1]) - one of the most significant Fréchet spaces. On the level of Fréchet spaces, the space $s$ is isomorphic to many classical spaces of smooth functions e.g. to the Schwartz space $\mathcal{S}(\mathbb{R})$ of smooth rapidly decreasing functions on the real line (which is a natural domain for the Fourier transform and differential operators with smooth coeficients), the space $C^{\infty}[-1,1]$ of smooth functions on the interval $[-1,1]$, the space $\mathcal{D}(K)$ of smooth functions with support contained in a compact set $K \subset \mathbb{R}^{n}, \operatorname{int}(K) \neq \emptyset$, or the space $C^{\infty}(M)$ of smooth functions on an arbitrary compact smooth manifold $M$. Finally, the space $s$ carries all the information about nuclear locally convex spaces. Indeed, the Kōmura-Kōmura theorem states that a locally convex space is nuclear if and only if it is isomorphic to some closed subspace of a suitable big cartesian product of the space $s$ (see [12, Th. 29.8]). Consequently, a Fréchet space is nuclear (i.e. a Fréchet space in which every unconditionally convergent series is already absolutely convergent) if and only if it is isomorphic to some closed subspace of $s^{\mathbb{N}}$ (see [12, Cor. 29.9]).

Replacing $s^{\prime}$ with the space $\mathcal{S}^{\prime}(\mathbb{R})$ of tempered distributions and $s$ with $\mathcal{S}(\mathbb{R})$, we still end up with the same Fréchet *-algebra - this time consisting of all linear and continuous operators acting from $\mathcal{S}^{\prime}(\mathbb{R})$ to $\mathcal{S}(\mathbb{R})$, where ${ }^{*}$-algebra operations are inherited from $L^{2}(\mathbb{R})$. In fact, for every Fréchet space $E$ isomorphic to $s$ and its topological dual $E^{\prime}$, the space $\mathcal{L}\left(E^{\prime}, E\right)$ (with appropriate ${ }^{*}$-algebra operations) of linear and continuous operators from $E^{\prime}$ to $E$ is a Fréchet *-algebra isomorphic to $\mathcal{L}\left(s^{\prime}, s\right)$ (for details see [3, Th. $1.10 \&$ Ex. 1.13] and [8, Th. 2.1]).

The algebra $\mathcal{L}\left(s^{\prime}, s\right)$ appears and plays a significant role in $K$-theory of Fréchet algebras (see Bhatt, Inoue and Ogi [1, Ex. 2.12], Cuntz [6, p. 144], [7, p. 64-65], Glöckner and Langkamp [10], Phillips [14, Def. 2.1]) and in $C^{*}$-dynamical systems (Elliot, Natsume and Nest [9, Ex. 2.6]). Recently, Piszczek obtained several rusults concerning closed ideals, automatic continuity, amenability, Jordan decomposition and Grothendieck-type inequality in $\mathcal{K}_{\infty}$ (see Piszczek [16, 17, 18, 19] and his forthcoming paper "The noncommutative Schwartz space is weakly amenable").

Recall that two bases $\left(x_{j}\right)_{j \in \mathbb{N}}$ and $\left(y_{j}\right)_{j \in \mathbb{N}}$ of a Fréchet space $X$ are called quasi-eqivalent if there is a permutation $\sigma: \mathbb{N} \rightarrow \mathbb{N}$, a sequence $\left(\lambda_{j}\right)_{j \in \mathbb{N}}$ of nonzero scalars and a Fréchet space isomorphism 
$T: X \rightarrow X$ such that $T x_{j}=\lambda_{j} y_{\sigma(j)}$ for $j \in \mathbb{N}$. It is still an open question - the so-called Quasiequivalence Conjecture - whether all bases in a nuclear Fréchet space are quasi-equivalent. This conjecture was posed in 1961 by Mityagin [13, §8.3]. There were many attempts to solve this problem (see [26] and references therein), e.g. Crone and Robinson [5] showed that in every nuclear Fréchet space with the so-called regular basis all bases are quasi-equivalent. In particular, all bases in $s$ are quasi-equivalent but the conjecture remains open for closed subspaces of $s$. As a consequence of Theorem 4, we obtain some characterization of closed subspaces of $s$ which have all bases quasiequivalent (Theorem 15). We hope that our characterization will shed some light on this long-standing conjecture.

\section{Preliminaries}

In what follows, $\mathbb{N}$ will denote the set of natural numbers $\{1,2, \ldots\}$ and $\mathbb{N}_{0}:=\mathbb{N} \cup\{0\}$.

By $e_{j}$ we denote the vector in $\mathbb{C}^{\mathbb{N}}$ whose $j$-th coordinate equals 1 and the others equal 0 .

By a Fréchet space we mean a complete metrizable locally convex space over $\mathbb{C}$ (we will not use locally convex spaces over $\mathbb{R}$ ). A Fréchet algebra is a Fréchet space which is an algebra with continuous multiplication. A Fréchet*-algebra is a Fréchet algebra with continuous involution.

We use the standard notation and terminology. All the notions from functional analysis are explained in [12].

We define the space of rapidly decreasing sequences as the Fréchet space

$$
s:=\left\{\xi=\left(\xi_{j}\right)_{j \in \mathbb{N}} \in \mathbb{C}^{\mathbb{N}}:|\xi|_{q}:=\left(\sum_{j=1}^{\infty}\left|\xi_{j}\right|^{2} j^{2 q}\right)^{1 / 2}<\infty \text { for all } q \in \mathbb{N}_{0}\right\}
$$

with the topology corresponding to the system $\left(|\cdot|_{q}\right)_{q \in \mathbb{N}_{0}}$ of norms. The strong dual of $s$ (i.e. the space of all continuous linear functionals on $s$ with the topology of uniform convergence on bounded subsets of $s$, see e.g. [12, Definition on p. 267]) can be identified with the space of slowly increasing sequences

$$
s^{\prime}:=\left\{\xi=\left(\xi_{j}\right)_{j \in \mathbb{N}} \in \mathbb{C}^{\mathbb{N}}:|\xi|_{q}^{\prime}:=\left(\sum_{j=1}^{\infty}\left|\xi_{j}\right|^{2} j^{-2 q}\right)^{1 / 2}<\infty \text { for some } q \in \mathbb{N}_{0}\right\} .
$$

It is an easy exercise to show that replacing in the above definition of $s$ and $s^{\prime}$ the $\ell_{2}$-norms by the corresponding sup-norms - as in (11) and (2) - or $\ell_{p}$-norms $(1 \leq p<\infty)$, we end up with the same sequence spaces.

Closed subspaces of the space $s$ can be characterized by the so-called property (DN) (see [12, Prop. $31.5])$.

Definition 1. A Fréchet space $X$ with a fundamental system $\left.\left(\|\cdot\|_{q}\right)_{q \in \mathbb{N}_{0}}\right)$ of seminorms has the property (DN) (see [12, Def. on p. 359]) if there is a continuous norm $\|\cdot\|$ on $X$ such that for all $q \in \mathbb{N}_{0}$ there is $r \in \mathbb{N}_{0}$ and $C>0$ such that

$$
\|x\|_{q}^{2} \leq C\|x\|\|x\|_{r}
$$

for all $x \in X$. The norm $\|\cdot\|$ is called a dominating norm.

We will also use the condition (DN) in the following equivalent form (see [12, Lemma 29.10]): there is a continuous norm $\|\cdot\|$ on $X$ such that for any $q \in \mathbb{N}_{0}$ and $\theta \in(0,1)$ there is $r \in \mathbb{N}_{0}$ and $C>0$ such that

for all $x \in X$.

$$
\|x\|_{q} \leq C\|x\|^{1-\theta}\|x\|_{r}^{\theta}
$$

The algebra of smooth operators is defined as the Fréchet ${ }^{*}$-algebra $\mathcal{L}\left(s^{\prime}, s\right)$ of continuous linear operators from $s^{\prime}$ to $s$ with the topology of uniform convergence on bounded sets in $s^{\prime}$. The ${ }^{*}$-algebra operations on $\mathcal{L}\left(s^{\prime}, s\right)$ are inherited from $\mathcal{B}\left(\ell_{2}\right)$ (note that every smooth operator is bounded on $\ell_{2}$ ). 
The algebra $\mathcal{L}\left(s^{\prime}, s\right)$ can be also seen as the algebra of matrices which are sometimes more handy; it is isomorphic, via the map $x \mapsto\left(\left\langle x e_{k}, e j\right\rangle\right)_{j, k \in \mathbb{N}}$, to the Fréchet ${ }^{*}$-algebra

$$
\mathcal{K}_{\infty}:=\left\{\left(x_{j, k}\right)_{j, k \in \mathbb{N}} \in \mathbb{C}^{\mathbb{N}^{2}}: \sup _{j, k \in \mathbb{N}}\left|x_{j, k}\right| j^{q} k^{q}<\infty \text { for all } q \in \mathbb{N}_{0}\right\}
$$

of rapidly decreasing matrices (with matrix multiplication and matrix complex conjugation). Therefore - when reading this paper - on can replace each " $\mathcal{L}\left(s^{\prime}, s\right)$ " with " $\mathcal{K}_{\infty}$ ". More details concerning $\mathcal{L}\left(s^{\prime}, s\right)$ can be find in the introductions of [2, 3, 4].

Definition 2. A matrix $A=\left(a_{j, q}\right)_{j \in \mathbb{N}, q \in \mathbb{N}_{0}}$ of nonnegative numbers such that

(i) for each $j \in \mathbb{N}$ there is $q \in \mathbb{N}_{0}$ such that $a_{j, q}>0$

(ii) $a_{j, q} \leq a_{j, q+1}$ for $j \in \mathbb{N}$ and $q \in \mathbb{N}_{0}$

is called a Köthe matrix. For $1 \leq p<\infty$ and a Köthe matrix $A$ we define the Köthe space

$$
\lambda^{p}(A):=\left\{\xi=\left(\xi_{j}\right)_{j \in \mathbb{N}} \in \mathbb{C}^{\mathbb{N}}:|\xi|_{\lambda^{p}(A), q}^{p}:=\sum_{j=1}^{\infty}\left|\xi_{j}\right|^{p} a_{j, q}^{p}<\infty \text { for all } q \in \mathbb{N}_{0}\right\}
$$

and for $p=\infty$

$$
\lambda^{\infty}(A):=\left\{\xi=\left(\xi_{j}\right)_{j \in \mathbb{N}} \in \mathbb{C}^{\mathbb{N}}:|\xi|_{\lambda \infty(A), q}:=\sup _{j \in \mathbb{N}}\left|\xi_{j}\right| a_{j, q}<\infty \text { for all } q \in \mathbb{N}_{0}\right\}
$$

with the locally convex topology given by the seminorms $\left(|\cdot|_{\lambda^{p}(A), q}\right)_{q \in \mathbb{N}_{0}}$ (see e.g. [12, Definition p. $326]$ ). For simplicity, we will also write $\lambda^{p}\left(a_{j, q}\right)$ (i.e. only the entries of the matix) instead of $\lambda^{p}(A)$.

All spaces $\lambda^{p}(A)$ are Fréchet spaces ([12, Lemma 27.1]). By the Dynin-Mityagin Theorem (see [12, Th. 28.12]) if $\lambda^{p}(A)$ is nuclear (i.e. for all $q$ there is $r$ such that $\sum_{j=1}^{\infty} \frac{a_{j, q}}{a_{j, r}}<\infty$ ), then the sequence of vectors $\left(e_{j}\right)_{j \in \mathbb{N}}$ is an absolute Schauder basis of $\lambda^{p}(A)$. We will also use the following result: $\lambda^{p}(A)$ is nuclear for some $1 \leq p \leq \infty$ if and only if $\lambda^{p}(A)=\lambda^{q}(A)$ as Fréchet spaces for all $1 \leq p, q \leq \infty([12$, Prop. 28.16]).

For Köthe matrices $A=\left(a_{j, q}\right)_{j \in \mathbb{N}, q \in \mathbb{N}_{0}}$ and $B=\left(b_{j, q}\right)_{j \in \mathbb{N}, q \in \mathbb{N}_{0}}$ such that

$$
\forall q \in \mathbb{N}_{0} \exists r \in \mathbb{N}_{0} \exists C>0 \forall j \in \mathbb{N} \quad a_{j, q} \leq C b_{j, r},
$$

we write $A \prec B$. If $A \prec B$ and $B \prec A$, then we write $A \sim B$. For a bijection $\sigma: \mathbb{N} \rightarrow \mathbb{N}, A_{\sigma}$ denotes the Köthe matrix $\left(a_{\sigma(j), q}\right)_{j \in \mathbb{N}, q \in \mathbb{N}_{0}}$ and, moreover, $A^{2}$ is by definition the Köthe matrix $\left(a_{j, q}^{2}\right)_{j \in \mathbb{N}, q \in \mathbb{N}_{0}}$.

By [15, Prop. 3.1], $\lambda^{1}(A)$ with pointwise multiplication is an algebra (and, clearly, if $\lambda^{1}(A)$ is nuclear so is $\lambda^{p}(A)$ for $\left.1<p \leq \infty\right)$ if and only if $A \prec A^{2}$; in this case $\lambda^{p}(A)$ is called a Köthe algebra.

Definition 3. We say that a Köthe space $\lambda^{p}(A)$ is closed under taking square root if it is a Köthe algebra and for each sequence $\left(\xi_{j}\right)_{j \in \mathbb{N}}$ in $\lambda^{p}(A)$ of nonnegative scalars, the sequence $\left(\sqrt{\xi_{j}}\right)_{j \in \mathbb{N}}$ belongs to $\lambda^{p}(A)$ as well.

\section{Commutative subalgebras of $\mathcal{L}\left(s^{\prime}, s\right)$}

Our main result - solving Problem (A) from Introduction - reads as follows:

Theorem 4. For a Köthe matrix $A=\left(a_{j, q}\right)_{j \in \mathbb{N}, q \in \mathbb{N}_{0}}$ the following assertions are equivalent:

(1) $\lambda^{2}(A)$ is isomorphic as a Fréchet ${ }^{*}$-algebra to some closed commutative ${ }^{*}$-subalgebra of $\mathcal{L}\left(s^{\prime}, s\right)$;

(2) there is a sequence $\left(P_{j}\right)_{j \in \mathbb{N}}$ of nonzero pairwise orthogonal self-adjoint projections belonging to $\mathcal{L}\left(s^{\prime}, s\right)$ such that $A \sim\left(\left\|P_{j}\right\|_{q}\right)_{j \in \mathbb{N}, q \in \mathbb{N}_{0}}$

(3) there is a basic sequence $\left(f_{j}\right)_{j \in \mathbb{N}}$ of $s$ which is at the same time an orthonormal sequence in $\ell_{2}$ such that $A \sim\left(\left|f_{j}\right|_{q}\right)_{j \in \mathbb{N}, q \in \mathbb{N}_{0}}$

(4) the matrix A satisfies the following conditions:

(i) $\forall q \in \mathbb{N}_{0} \exists r \in \mathbb{N}_{0} \quad \sum_{j=1}^{\infty} \frac{a_{j, q}}{a_{j, r}}<\infty$,

(ii) $\exists p \in \mathbb{N}_{0} \exists C>0 \forall j \in \mathbb{N} \quad a_{j, p} \geq \frac{1}{C}$, 
(iii) $A^{2} \prec A$;

(5) the matrix $A$ satisfies the following conditions:

(i) $\forall q \in \mathbb{N}_{0} \exists r \in \mathbb{N}_{0} \quad \sum_{j=1}^{\infty} \frac{a_{j, q}}{a_{j, r}}<\infty$,

(ii) $\exists p \in \mathbb{N}_{0} \forall q \in \mathbb{N}_{0} \exists r \in \mathbb{N}_{0}, C>0 \forall j \in \mathbb{N} \quad a_{j, q}^{2} \leq C a_{j, p} a_{j, r}$,

(iii) $A \sim A^{2}$;

(6) $\lambda^{2}(A)$ is a nuclear biprojective Köthe algebra with the property (DN);

(7) $\lambda^{2}(A)$ is isomorphic as a Fréchet space to a closed subspace of $s$ and it is closed under taking square roots;

(8) $\lambda^{2}(A)$ is a nuclear Fréchet space with $\|\cdot\|_{\ell_{2}}$ as a dominating norm.

Remark 5. By [2, Th. 4.8], every infinite-dimensional closed commutative ${ }^{*}$-subalgebra of $\mathcal{L}\left(s^{\prime}, s\right)$ is isomorphic as a Fréchet ${ }^{*}$-algebra to some Köthe sequence algebra, and thus the above theorem characterizes in fact all infinite-dimensional closed commutative ${ }^{*}$-subalgebras of $\mathcal{L}\left(s^{\prime}, s\right)$.

Remark 6. Homology theory is out of the mainstream of this paper, and therefore we only recall that the a Fréchet algebra $A$ is called biprojective if the product map $\pi: A \hat{\otimes} A \rightarrow A, a \otimes b \mapsto a b$, has a right inverse in the category of $A$-bimodules (see also [11, Def. IV.5.1]). The characterization of biprojectivity for Köthe algebras in terms of the corresponding Köthe matrices is due to Pirkovskii [15, Th. 5.9].

The following theorem leads to the solution of Problem (B) (see Introduction).

Theorem 7. For a Köthe matrix $A=\left(a_{j, q}\right)_{j \in \mathbb{N}, q \in \mathbb{N}_{0}}$ the following assertions are equivalent:

(1) $\lambda^{2}(A)$ is isomorphic as a Fréchet space to some closed commutative ${ }^{*}$-subalgebra of $\mathcal{L}\left(s^{\prime}, s\right)$;

(2) the matrix $A$ satisfies the following conditions:

(i) $\forall q \in \mathbb{N}_{0} \exists r \in \mathbb{N}_{0} \quad \sum_{j=1}^{\infty} \frac{a_{j, q}}{a_{j, r}}<\infty$,

(ii) $\exists p \in \mathbb{N}_{0} \forall q \in \mathbb{N}_{0} \exists r \in \mathbb{N}_{0}, C>0 \forall j \in \mathbb{N} \quad a_{j, q}^{2} \leq C a_{j, p} a_{j, r}$;

(3) $\lambda^{2}(A)$ is a nuclear Fréchet space with the property (DN).

(4) $\lambda^{2}(A)$ is a Fréchet space isomorphic to a closed subspace of $s$.

Proof. It is well known that a Köthe space $\lambda^{2}(A)$ is nuclear and has the property (DN) if and only if $A$ satifies condtions (i) and (ii), respectively. This shows $(1) \Rightarrow(2) \Rightarrow(3)$, because $\mathcal{L}\left(s^{\prime}, s\right) \cong s$ as Fréchet spaces and nuclearity together with the property (DN) are inherited by closed subspaces.

In order to prove $(3) \Rightarrow(1)$, choose $p_{0} \in \mathbb{N}_{0}$ for which $|\cdot|_{\lambda^{2}(A), p_{0}}$ is dominating norm on $\lambda^{2}(A)$ and define the Köthe matrix $B:=\left(a_{j, q} / a_{j, p_{0}}\right)_{j \in \mathbb{N}, q \in \mathbb{N}_{0}}$. Let $T: \lambda^{2}(B) \rightarrow \lambda^{2}(A), T \xi:=\left(\xi_{j} / a_{j, p_{0}}\right)_{j \in \mathbb{N}}$. Then, for $\xi \in \lambda^{2}(B)$ and $q \in \mathbb{N}_{0}$, we have $|T \xi|_{\lambda^{2}(A), q}=|\xi|_{\lambda^{2}(B), q}$, and thus $T$ is an isomorphism of Fréchet spaces. Moreover, for $q \in \mathbb{N}_{0}$ there is $r \in \mathbb{N}_{0}$ and $C>0$ such that

$$
|\xi|_{\lambda^{2}(B), q}^{2}=|T \xi|_{\lambda^{2}(A), q}^{2} \leq C|T \xi|_{\lambda^{2}(A), p_{0}}|T \xi|_{\lambda^{2}(A), r}=C|| \xi||_{\ell_{2}}|\xi|_{\lambda^{2}(B), r}
$$

$\xi \in \lambda^{2}(B)$. Therefore, $\|\cdot\|_{\ell_{2}}$ is a dominating norm on $\lambda^{2}(B)$, and the conclusion follows from Theorem 4 (implication $(8) \Rightarrow(1)$ ).

Finally, equivalence $(3) \Leftrightarrow(4)$ follows directly from [12, Prop. 31.5].

As a direct consequence of Theorems 4, 7 and [12, Cor. 28.13, Prop. 28.16, Prop. 31.5] we also get the following characterization.

Corollary 8. For a Fréchet space $E$ the following assertions are equivalent:

(1) $E$ is isomorphic as a Fréchet space to some closed commutative ${ }^{*}$-subalgebra of $\mathcal{L}\left(s^{\prime}, s\right)$;

(2) $E$ is isomorphic as a Fréchet space to a Köthe space $\lambda^{2}\left(\left|f_{j}\right|_{q}\right)$ for some basic sequence $\left(f_{j}\right)_{j \in \mathbb{N}}$ of $s$ which is at the same time an orthonormal sequence in $\ell_{2}$;

(3) $E$ is nuclear, has a basis and the property (DN);

(4) $E$ is isomorphic as a Fréchet space to some closed subspace of $s$ with basis. 
Now, we shall provide the proof of Theorem 4. We start with some lemmas involving short exact sequences of Fréchet-Hilbert spaces.

Lemma 9. Let

$$
0 \longrightarrow E \stackrel{j}{\longrightarrow} F \stackrel{q}{\longrightarrow} G \longrightarrow 0
$$

be a short exact sequence of Fréchet-Hilbert spaces. Let $\|\cdot\|_{E}$ be a continuous Hilbert norm on $E$ and let $\|\cdot\|_{G}$ be a continuous norm on $G$. Then there is a continuous Hilbert norm $\|\cdot\|_{F}$ on $F$ with

$(\alpha)\|x\|_{E} \sim\|j(x)\|_{F}$ for $x \in E$;

( $\beta)\|q(x)\|_{G} \leq \inf _{y \in \operatorname{ker} q}\|x-y\|_{F}$ for $x \in F$.

Proof. Without lost of generality we may assume that $E$ is a closed subspace of $F$ and $j(x)=x$ for $x \in E$. By [12, Remark 22.8], there is a continuous Hilbert norm $\|\cdot\|_{1}$ on $F$ with $\|x\|_{E} \leq\|x\|_{1}$ for $x \in E$. Moreover, by the continuity of $q$, there is a continuous Hilbert norm $\|\cdot\|_{2}$ on $F$ such that

$$
\|q(x)\|_{G} \leq \inf _{z \in E}\|x-z\|_{2}
$$

for $x \in F$. Then $\|\cdot\|:=\left(\|\cdot\|_{1}^{2}+\|\cdot\|_{2}^{2}\right)^{1 / 2}$ is a continuous Hilbert norm on $F$ such that

(i) $\|x\|_{E} \leq\|x\|$ for $x \in E$;

(ii) $\|q(x)\|_{G} \leq \inf _{z \in E}\|x-z\|$ for $x \in F$.

Define $\|\cdot\|_{F}$, by

$$
\|x\|_{F}^{2}=\inf _{z \in E}\left(\|z\|_{E}^{2}+\|x-z\|^{2}\right)
$$

for $x \in F$.

To show condition $(\alpha)$, let us fix $x \in E$. Taking $z:=x$ in the infimum, we get

$$
\|x\|_{F} \leq\|x\|_{E} .
$$

Moreover, for $z \in E$, we obtain

$$
\|x\|_{E}^{2} \leq\left(\|z\|_{E}+\|x-z\|_{E}\right)^{2}=\|z\|_{E}^{2}+\|x-z\|_{E}^{2}+2\|z\|_{E}\|x-z\|_{E} .
$$

If $\|z\|_{E} \leq\|x-z\|_{E}$, then $\|z\|_{E}\|x-z\|_{E} \leq\|x-z\|_{E}^{2}$. Otherwise, $\|z\|\left\|_{E}\right\| x-z\left\|_{E} \leq\right\| z \|_{E}^{2}$ so, by (i),

$$
\|x\|_{E}^{2} \leq 3\left(\|z\|_{E}^{2}+\|x-z\|_{E}^{2}\right) \leq 3\left(\|z\|_{E}^{2}+\|x-z\|^{2}\right) .
$$

In consequence,

$$
\|x\|_{E} \leq \sqrt{3} \inf _{z \in E}\left(\|z\|_{E}^{2}+\|x-z\|^{2}\right)^{1 / 2}=\sqrt{3}\|x\|_{F},
$$

and thus condition $(\alpha)$ if fulfilled. By (ii), for $x \in F$, we get

$$
\begin{aligned}
\|q(x)\|_{G}^{2} & \leq \inf _{z \in E}\|x-z\|^{2}=\inf _{z_{1} \in E} \inf _{z_{2} \in E}\left(\left\|z_{2}\right\|_{E}^{2}+\left\|x-z_{1}\right\|^{2}\right)=\inf _{z_{1}, z_{2} \in E}\left(\left\|z_{2}\right\|_{E}^{2}+\left\|x-\left(z_{1}+z_{2}\right)\right\|^{2}\right) \\
& =\inf _{z_{1} \in E} \inf _{z_{2} \in E}\left(\left\|z_{2}\right\|_{E}^{2}+\left\|\left(x-z_{1}\right)-z_{2}\right\|^{2}\right)=\inf _{z \in E}\|x-z\|_{F}^{2},
\end{aligned}
$$

which yields condition $(\beta)$.

Now define a Hilbert norm $\|(\cdot, \cdot)\|_{H}$ on $H:=E \times F$ by

$$
\|(x, y)\|_{H}^{2}:=\|x\|_{E}^{2}+\|y\|^{2} .
$$

Let

$$
R:\left(H,\|(\cdot, \cdot)\|_{H}\right) \rightarrow F, \quad(x, y) \mapsto x+y
$$

and let

$$
Q:\left(H,\|\cdot\|_{H}\right) \rightarrow\left(H / \operatorname{ker} R,\|\cdot\|_{H / \operatorname{ker} R}\right)
$$


be the quotient map. Then

$$
\begin{aligned}
\|Q(x, y)\|_{H / \operatorname{ker} R} & =\inf _{\left(x^{\prime}, y^{\prime}\right) \in \operatorname{ker} R}\left\|(x, y)-\left(x^{\prime}, y^{\prime}\right)\right\|_{H}=\inf _{x^{\prime} \in E}\left\|(x, y)-\left(x^{\prime},-x^{\prime}\right)\right\|_{H} \\
& =\inf _{x^{\prime} \in E}\left(\left\|x-x^{\prime}\right\|_{E}^{2}+\left\|y+x^{\prime}\right\|^{2}\right)^{1 / 2}=\inf _{z \in E}\left(\|z\|_{E}^{2}+\|(x+y)-z\|^{2}\right)^{1 / 2} \\
& =\|x+y\|_{F} .
\end{aligned}
$$

In particular

$$
\|\cdot\|_{F}=\|Q(0, \cdot)\|_{H / \operatorname{ker} R}
$$

hence $\|\cdot\|_{F}$ is a seminorm. Moreover, since $\|\cdot\|_{F} \leq\|\cdot\|$, it follows that $\|\cdot\|_{F}$ is continuous.

Now assume that $\|x\|_{F}=0$ for some $x \in F$. Then, by condition $(\beta),\|q(x)\|_{G} \leq\|x\|_{F}=0$, and since $\|\cdot\|_{G}$ is a norm, we have $x \in \operatorname{ker} q=E$. Moreover, by (4), $\|x\|_{E} \leq \sqrt{3}\|x\|_{F}=0$, but $\|\cdot\|_{E}$ is a norm, so $x=0$. This shows that $\|\cdot\|_{F}$ is a norm, and therefore, by (5), $\|\cdot\|_{H / \operatorname{ker} R}$ is a norm as well (i.e. $\operatorname{ker} R$ is a closed subspace of $H$ - see e.g. [12, Lemma 5.10]). Since the quotient norm induced by a Hilbert norm is a Hilbert norm, the norm $\|\cdot\|_{H / \operatorname{ker} R}$ is hilbertian (see e.g. [20, p. 44]). Therefore, by ([6), $\|\cdot\|_{F}$ satisfies the parallelogram law, hence $\|\cdot\|_{F}$ is a Hilbert norm.

An easy proof of our next lemma is left to the reader.

Lemma 10. Let us consider the diagram

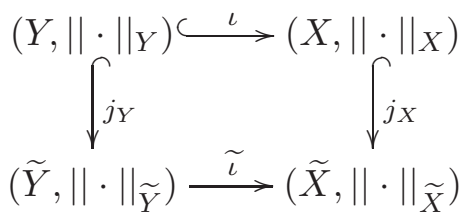

where

- $\left(X,\|\cdot\|_{X}\right)$ and $\left(Y,\|\cdot\|_{Y}\right)$ are normed spaces such that $Y$ is a closed subspace of $\left(X,\|\cdot\|_{X}\right)$ and $\|\cdot\|_{Y} \sim\|\cdot\|_{X \mid Y}$

- $\left(\widetilde{Y},\|\cdot\|_{\widetilde{Y}}\right)$ and $\left(\widetilde{X},\|\cdot\|_{\widetilde{X}}\right)$ are the completions of $\left(Y,\|\cdot\|_{Y}\right)$ and $\left(X,\|\cdot\|_{X}\right)$, respectively;

- $\iota, j_{Y}$ and $j_{X}$ are the canonical inclusions;

- $\widetilde{\iota}$ is the continuous linear extensions of the map $j_{X} \circ \iota: Y \rightarrow \widetilde{X}$.

Then $\widetilde{\iota}(\widetilde{Y})$ is a closed subspace of $\left(\widetilde{X},\|\cdot\|_{\tilde{X}}\right)$.

Lemma 11. Let

$$
0 \longrightarrow E \stackrel{j}{\longrightarrow} F \stackrel{q}{\longrightarrow} G \longrightarrow 0
$$

be a short exact sequence of Fréchet-Hilbert spaces. Let $\|\cdot\|_{E}$ be a continuous Hilbert norm on $E$ and let us assume that $G$ has a continuous norm. Then there is a continuous Hilbert norm $\|\cdot\|$ on $F$ such that $\|j(x)\|=\|x\|_{E}$ for $x \in E$.

Proof. Without lost of generality we may assume that $E$ is a closed subspace of $F$ and $j(x)=x$ for $x \in E$. Let $|\cdot|_{G}$ be a continuous norm on $G$. By Lemma 9, there is a continuous Hilbert norm $\|\cdot\|_{F}$ on $F$ such that

$(\alpha)\|\cdot\|_{E} \sim\|\cdot\|_{F \mid E}$ for $x \in E$

(ß) $|q(x)|_{G} \leq \inf _{y \in \operatorname{ker} q}\|x-y\|_{F}=:\|x\|_{G}$ for $x \in F$. 
Consider the diagram

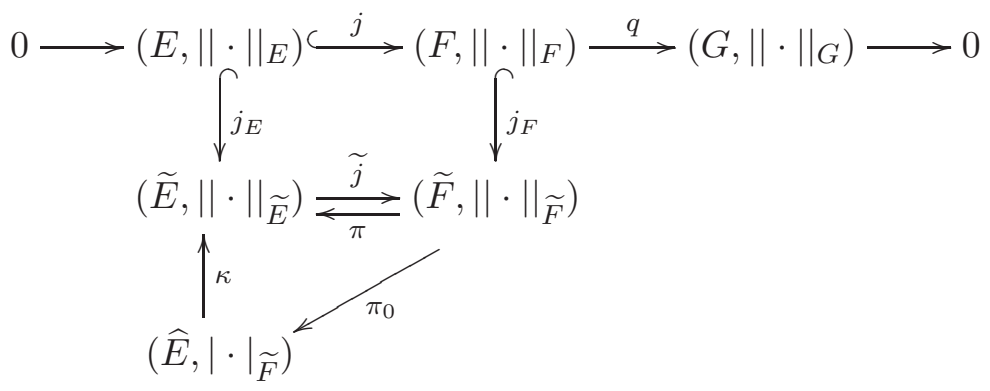

where

- $\left(\widetilde{E},\|\cdot\|_{\widetilde{E}}\right)$ and $\left(\widetilde{F},\|\cdot\|_{\widetilde{F}}\right)$ are the completions of $\left(E,\|\cdot\|_{E}\right)$ and $\left(F,\|\cdot\|_{F}\right)$, respectively;

- $j_{E}$ and $j_{F}$ are the canonical inclusions;

- $\widetilde{j}$ is the continuous extensions of the map $j_{F} \circ j: E \rightarrow \widetilde{F}$.

Since, by $(\beta),\|\cdot\|_{G}$ is a norm, $E$ is a closed subspace of $\left(F,\|\cdot\|_{F}\right)$, and thus, by Lemma 10, $\widehat{E}:=\widetilde{j}(\widetilde{E})$ is a closed subspace of the Hilbert space $\left(\widetilde{F},\|\cdot\|_{\widetilde{F}}\right)$. Then $\pi_{0}$ is defined to be the orthogonal projections onto $\widehat{E}, \kappa$ is the inverse of $\widetilde{\iota}$ on $\widehat{E}$ and $\pi:=\kappa \circ \pi_{0}$.

Let us define

$$
\|x\|^{2}:=\left\|\left(\pi \circ j_{F}\right)(x)\right\|_{\widetilde{E}}^{2}+\|q(x)\|_{G}^{2}
$$

for $x \in F$. Clearly, $\|\cdot\|$ is a continuous seminorm on $F$. Next, for $x \in E$, we get

$$
\|x\|=\left\|\left(\pi \circ j_{F}\right)(x)\right\|_{\widetilde{E}}=\left\|j_{E}(x)\right\|_{\widetilde{E}}=\|x\|_{E},
$$

hence $\|\cdot\|_{\mid E}=\|\cdot\|_{E}$. For $x \in F$ with $\|x\|=0$ we have $\|q(x)\|_{G}=0$, and thus $x \in E$. Then we have also $\|x\|_{E}=\|x\|=0$ so $x=0$. This shows that $\|\cdot\|$ is a norm.

Finally, since $\|\cdot\|_{\widetilde{E}}$ and $\|\cdot\|_{G}$ are Hilbert norms, $\|\cdot\|$ is a Hilbert norm as well.

The proof of the following lemma is a slight modification of the Vogt's proof of [23, Lemma 3.1]. For the convenience of the reader we present here the full argument.

Lemma 12. Let

$$
0 \longrightarrow E \stackrel{j}{\longrightarrow} F \stackrel{q}{\longrightarrow} G \longrightarrow 0
$$

be a short exact sequence of Fréchet-Hilbert spaces. Let $\|\cdot\|_{E}$ be dominating Hilbert norm on $E$ and let us assume that $G$ has the property $(\mathrm{DN})$. Then there is a dominating Hilbert norm $\|\cdot\|$ on $F$ such that $\|j(x)\|=\|x\|_{E}$ for $x \in E$.

Proof. Without lost of generality we may assume that $E$ is a closed subspace of $F$ and $j(x)=x$ for $x \in E$. By Lemma 11, there is continuous Hilbert norm $\|\cdot\|_{F}$ on $F$ such that $\|\cdot\|_{F \mid E}=\|\cdot\|_{E}$ and, by assumption, there is a dominating Hilbert norm $\|\cdot\|_{G}$ on $G$. Define

$$
\|x\|^{2}:=\|x\|_{F}^{2}+\|q x\|_{G}^{2}
$$

for $x \in F$ and let $U_{0}:=\{x \in E:\|x\|<1\}$. Then, clearly, $\|\cdot\|$ is a continuous Hilbert norm on $F$. We shall show that $\|\cdot\|$ is a dominating norm.

For an absolutely convex zero neighborhood $U$ in $F$, let $\|\cdot\|_{U}$ denote the Minkowski functional for $U$ and let $\|\cdot\|_{\widehat{U}}: G \rightarrow[0, \infty)$ be defined by $\|q x\|_{\widehat{U}}:=\inf \left\{\|x+y\|_{U}: y \in E\right\}$ for $x \in F$.

Since $\|\cdot\|_{E}$ is a dominating norm on $E$, for each absolutely convex zero neighborhood $U$ in $F$ there is an absolutely convex zero neighborhood $W$ in $F$ with $W \subseteq U \cap U_{0}$ such that

$$
\|z\|_{U} \leq\|z\|_{E}^{2 / 3}\|z\|_{W}^{1 / 3},
$$


for all $z \in E$. Since $\|\cdot\|_{G}$ is a dominating norm on $G$, there is an absolutely convex zero neighborhood $V$ in $F$ with $V \subseteq W$ such that

$$
\|q x\|_{\widehat{W}} \leq\|q x\|_{G}^{3 / 4}\|q x\|_{\widehat{V}}^{1 / 4} \leq\|x\|^{3 / 4}\|x\|_{V}^{1 / 4}
$$

for all $x \in F$.

Let us fix $x \in F$ and $\varepsilon>0$. By the very definition of the norm $\|\cdot\|_{\widehat{W}}$, there is $y \in F$ such that

$$
q x=q y \quad \text { and } \quad\|y\|_{W} \leq\|q x\|_{\widehat{W}}+\varepsilon,
$$

and thus (8) yields

$$
\|y\|_{W} \leq\|x\|^{3 / 4}\|x\|_{V}^{1 / 4}+\varepsilon
$$

Hence, for $z:=x-y$, we have

$$
\|z\|_{E} \leq\|x\|_{F}+\|y\|_{F} \leq\|x\|+\|y\|_{W} \leq\|x\|+\|x\|^{3 / 4}\|x\|_{V}^{1 / 4}+\varepsilon,
$$

and since

$$
\|x\|=\|x\|^{3 / 4}\|x\|^{1 / 4} \leq\|x\|^{3 / 4}\|x\|_{V}^{1 / 4}
$$

it follows that

$$
\|z\|_{E} \leq 2\|x\|^{3 / 4}\|x\|_{V}^{1 / 4}+\varepsilon
$$

Moreover, by (9),

$$
\|z\|_{W} \leq\|x\|_{W}+\|y\|_{W} \leq\|x\|_{V}+\|x\|^{3 / 4}\|x\|_{V}^{1 / 4}+\varepsilon \leq 2\|x\|_{V}+\varepsilon .
$$

Now, since for $a, b \geq 0$ we have $(a+b)^{2 / 3} \leq 3\left(a^{2 / 3}+b^{2 / 3}\right)$, it follows from (7) and (10) that

$$
\begin{aligned}
\|z\|_{U} & \leq\left(2\|x\|^{3 / 4}\|x\|_{V}^{1 / 4}+\varepsilon\right)^{2 / 3}\left(2\|x\|_{V}+\varepsilon\right)^{1 / 3} \\
& \leq\left[3\left(2\|x\|^{3 / 4}\|x\|_{V}^{1 / 4}\right)^{2 / 3}+\varepsilon^{2 / 3}\right]\left[\left(2\|x\|_{V}\right)^{1 / 3}+\varepsilon^{1 / 3}\right] \\
& =\left(3 \sqrt[3]{4}\|x\|^{1 / 2}\|x\|_{V}^{1 / 6}+3 \varepsilon^{2 / 3}\right)\left(\sqrt[3]{2}\|x\|_{V}^{1 / 3}+\varepsilon^{1 / 3}\right)=6\|x\|^{1 / 2}\|x\|_{V}^{1 / 2}+h(\varepsilon),
\end{aligned}
$$

where $h(\varepsilon) \searrow 0$ when $\varepsilon \searrow 0$.

Next, by (9),

$$
\|y\|_{U} \leq\|y\|_{W} \leq\|x\|^{3 / 4}\|x\|_{V}^{1 / 4}+\varepsilon=\|x\|^{1 / 2}\|x\|_{V}^{1 / 2}\left(\frac{\|x\|}{\|x\|_{V}}\right)^{1 / 4}+\varepsilon \leq\|x\|^{1 / 2}\|x\|_{V}^{1 / 2}+\varepsilon .
$$

Consequently, by (11),

$$
\|x\|_{U} \leq\|y\|_{U}+\|z\|_{U} \leq 7\|x\|^{1 / 2}\|x\|_{V}^{1 / 2}+h(\varepsilon)+\varepsilon,
$$

and finally, since $h(\varepsilon)+\varepsilon$ can be chosen arbitrary small, we have

$$
\|x\|_{U}^{2} \leq 49\|x\|\|x\|_{V}
$$

which shows that $\|\cdot\|$ is a dominating norm on $F$.

Corollary 13. Let $E$ be a closed subspace of s such that $s / E$ has a dominating norm. Then for every dominating Hilbert norm $\|\cdot\|_{E}$ on $E$ there is a dominating Hilbert norm $\|\cdot\|$ on $s$ such that the restriction of $\|\cdot\|$ to $E$ is equal to $\|\cdot\|_{E}$. 
Proof. The results follows by applying Lemma 12 to the canonical short exact sequence

$$
0 \longrightarrow E \hookrightarrow s \longrightarrow s / E \longrightarrow 0 \text {. }
$$

Corollary 13 together with some results of Vogt ([25, Cor. 7.6], [22, Prop. 3.3(a)]) allows to give a stronger version of the well-kown theorem of Vogt characterizing closed subspaces of $s$ in terms of nuclearity and the property (DN) (see [21, Satz 1.7] or [12, Prop. 31.5]).

Theorem 14. For a Fréchet space $X$ and a seminorm $\|\cdot\|: X \rightarrow[0, \infty)$ the following assertions are equivalent:

(1) there is a topological embedding $V: X \hookrightarrow s$ such that $\|V x\|_{\ell_{2}}=\|x\|$ for all $x \in X$;

(2) $X$ is nuclear and $\|\cdot\|$ is a dominating Hilbert norm on $X$.

Proof. $(\Rightarrow)$ By assumption, $X$ is isomorphic to a closed subspace of $s$, so it is nuclear. Moreover, since $\|\cdot\|_{\ell_{2}}$ is a dominating Hilbert norm on $s$, it is easily seen that $\|\cdot\|$ is a dominating Hilbert norm on $X$.

$(\Leftarrow)$ Since $X$ is a nuclear Fréchet space with the property (DN), by Theorem [12, Prop. 31.5], it is isomorphic to some closed subspace $E$ of $s$. Without lost of generality we may assume that $s / E$ has (DN). Indeed, by [22, Prop. 3.3(a)], for an arbitrary closed subspace $E_{0}$ of $s$ isomorphic to $X$, there is a closed subspace $F_{0}$ of $s$ and an exact sequence

$$
0 \longrightarrow E_{0} \stackrel{\iota_{0}}{\longrightarrow} s \stackrel{q_{0}}{\longrightarrow} F_{0} \longrightarrow 0
$$

with continuous linear maps. Then $q_{0}$ induces an isomorphism $\overline{q_{0}}: s /$ ker $q_{0} \rightarrow F_{0}$ (see [12, Prop. $22.11]$ ), and thus

$$
s / \iota_{0}\left(E_{0}\right)=s / \operatorname{ker} q_{0} \cong F_{0} .
$$

Hence $E:=\iota_{0}\left(E_{0}\right)$ is a closed subspace of $s$ such that $s / E$ has (DN), and by the open mapping theorem $E \cong E_{0}$.

Let $T: X \rightarrow E$ be an isomorphism and set $\|x\|_{E}:=\left\|T^{-1} x\right\|$ for $x \in E$. An easy calculation shows that $\|\cdot\|_{E}$ is a dominating Hilbert norm on $E$. Hence, by Corollary 13, there is a dominating Hilbert norm $\|\cdot\|_{s}$ on $s$ such that $\|\cdot\|_{s \mid E}=\|\cdot\|_{E}$. Moreover, by [25, Cor. 7.6], there is an automorphism $U$ of $s$ such that $\|U x\|_{\ell_{2}}=\|x\|_{s}$ for $x \in s$. Now, for $V:=U \circ T$ and $x \in X$, we get

$$
\|V x\|_{\ell_{2}}=\|U(T x)\|_{\ell_{2}}=\|T x\|_{s}=\|T x\|_{E}=\|x\|,
$$

which proves the theorem.

Finally, we are ready to proof our main result.

Proof of Theorem 4. (3) $\Rightarrow(1)$ : We should only note that, by [4, Prop. 4.2], $\lambda^{2}(A) \cong \lambda^{\infty}\left(\left|f_{j}\right|_{q}\right)$ as a Fréchet ${ }^{*}$-algebras and $\lambda^{\infty}\left(\left|f_{j}\right|_{q}\right)$ is isomorphic as a Fréchet ${ }^{*}$-algebra to the closed ${ }^{*}$-subalgebra of $\mathcal{L}\left(s^{\prime}, s\right)$ generated by the sequence $\left(f_{j} \otimes f_{j}\right)_{j \in \mathbb{N}}$.

$(1) \Rightarrow(2)$ : Let $E$ be a closed commutative ${ }^{*}$-subalgebra of $\mathcal{L}\left(s^{\prime}, s\right)$ isomorphic to the Fréchet ${ }^{*}$ algebra $\lambda^{2}(A)$. By [2, Th. 4.8] and the nuclearity of $E$, there is a sequence $\left(Q_{j}\right)_{j \in \mathbb{N}}$ of nonzero pairwise orthogonal self-adjoint projections belonging to $\mathcal{L}\left(s^{\prime}, s\right)$ such that $\lambda^{2}(A) \cong E \cong \lambda^{2}\left(\left\|Q_{j}\right\|_{q}\right)$ as Fréchet ${ }^{*}$-algebras. Therefore, according to [4, Prop. 4.2], there is a bijection $\sigma: \mathbb{N} \rightarrow \mathbb{N}$ such that for $P_{j}:=Q_{\sigma(j)}(j \in \mathbb{N})$, we have $A \sim\left(\left\|P_{j}\right\|_{q}\right)_{j \in \mathbb{N}, q \in \mathbb{N}_{0}}$.

$(2) \Rightarrow(4)$ : By [2, Prop. 4.7] and [12, Lemma 27.25], $\lambda^{2}\left(\left\|P_{j}\right\|_{q}\right)$ is isomorphic to the closed *subalgebra of $\mathcal{L}\left(s^{\prime}, s\right)$ generated by the sequence $\left(P_{j}\right)_{j \in \mathbb{N}}$. Therefore, the space $\lambda^{2}(A)$ (which is isomorphic as a Fréchet ${ }^{*}$-algebra to $\left.\lambda^{2}\left(\left\|P_{j}\right\|_{q}\right)\right)$ is nuclear, and consequently, the matrix $A$ satisfies condition (i). Moreover, it is easy to check that the matrix $\left(\left\|P_{j}\right\|_{q}\right)_{j \in \mathbb{N}, q \in \mathbb{N}_{0}}$ fulfils conditions (ii) and (iii), and thus (ii) and (iii) are also satisfied by the matrix $A$ (apply [4, Prop. 4.2]).

$(4) \Rightarrow(5)$ : By conditions (ii) and (iii), we get

$$
a_{j, r} \leq C a_{j, p} a_{j, r} \leq C a_{j, \max \{p, r\}}^{2}
$$


and

$$
a_{j, q}^{2} \leq C_{q} a_{j, r} \leq C C_{q} a_{j, p} a_{j, r}
$$

with appropriate quantifiers and constants.

$(5) \Leftrightarrow(6)$ : It is well known that condtions (i) and (ii) are equivalent to nuclearity and the property (DN), respectively. By [15, Prop. 3.1, Th. 5.9], $\lambda^{\infty}(A)$ is a biprojective Köthe algebra if and only if $A$ satifies condition (iii).

$(5) \Leftrightarrow(7)$ : By [12, Prop. 31.5], conditions (i) and (ii) are equivalent to the fact that $\lambda^{2}(A)$ is isomorphic to a closed subspace of $s$ and, by [15, Prop. 3.1], $\lambda^{\infty}(A)$ is an algebra if and only if $A \prec A^{2}$. Moreover, by [15, Lemmas 7.7 and 7.9], conditions (i) and (iii) imply that the square root of each nonnegative element of $\lambda^{\infty}(A)$ belongs to $\lambda^{\infty}(A)$. On the other hand, if $\lambda^{2}(A)$ is closed under taking square root and nuclear (note that closed subspaces of $s$ are nuclear) then, by [12, Prop. 28.16], $\lambda^{2}(A) \subset \lambda^{2}\left(A^{2}\right)$. Consequently, by the closed graph theorem, the identity map from $\lambda^{2}(A)$ to $\lambda^{2}\left(A^{2}\right)$ is continuous, and hence $A^{2} \prec A$.

$(5) \Rightarrow(8)$ : Nuclearity is guaranteed by condition (i). For an index $p$ from condition (ii) take, according to (i), an index $r$ and a constant $C_{0}>0$ such that $\sum_{j=1}^{\infty} \frac{a_{j, p}}{a_{j, r}}<C_{0}$. By (ii) and (iii) (more precisely by $A \prec A^{2}$ ), there are $q_{1}, q_{2}, q_{3} \in \mathbb{N}_{0}$ and constants $C_{1}, C_{2}, C_{3}>0$ such that

$$
a_{j, r} \leq C_{1} a_{j, q_{1}}^{2} \leq C_{2} a_{j, p} a_{j, q_{2}} \leq C_{3} a_{j, p} a_{j, q_{3}}^{2} .
$$

Then for $\xi \in \lambda^{2}(A)$ we obtain

$$
\|\xi\|_{\ell_{2}} \leq C_{3} \sum_{j=1}^{\infty}\left|\xi_{j}\right|^{2} \frac{a_{j, p} a_{j, q_{3}}^{2}}{a_{j, r}} \leq C_{3} \sum_{j=1}^{\infty} \frac{a_{j, p}}{a_{j, r}} \cdot \sup _{j \in \mathbb{N}}\left|\xi_{j}\right|^{2} a_{j, q_{3}}^{2} \leq C_{0} C_{3}|\xi|_{\lambda^{2}(A), q_{3}}^{2}<\infty,
$$

and thus $\|\cdot\|_{\ell_{2}}$ is a norm on $\lambda^{2}(A)$.

Moreover, the Cauchy-Schwartz inequality and condition (iii) (more precisely relation $A^{2} \prec A$ ) imply that for all $q \in \mathbb{N}_{0}$ there are $r \in \mathbb{N}_{0}$ and $C>0$ such that

$$
\begin{aligned}
|\xi|_{\lambda^{2}(A), q}^{2} & :=\sum_{j \in \mathbb{N}}\left|\xi_{j}\right|^{2} a_{j, q}^{2} \leq C\left(\sum_{j \in \mathbb{N}}\left|\xi_{j}\right|^{2}\right)^{1 / 2} \cdot\left(\sum_{j \in \mathbb{N}}\left|\xi_{j}\right|^{2} a_{j, q}^{4}\right)^{1 / 2} \leq C\|\xi\|_{\ell_{2}}\left(\sum_{j \in \mathbb{N}}\left|\xi_{j}\right|^{2} a_{j, r}^{2}\right)^{1 / 2} \\
& =C \| \xi||_{\ell_{2}}|\xi|_{\lambda^{2}(A), r}
\end{aligned}
$$

which shows that $\|\cdot\|_{\ell_{2}}$ is a dominating norm on $\lambda^{2}(A)$.

$(8) \Rightarrow(3)$ : By Theorem 14, there is a topological embedding $V: \lambda^{2}(A) \hookrightarrow s$ such that $\|V \xi\|_{\ell_{2}}=\|\xi\|_{\ell_{2}}$ for all $\xi \in \lambda^{2}(A)$. Set $f_{j}:=V e_{j}$ for $j \in \mathbb{N}$. Then $\left(f_{j}\right)_{j \in \mathbb{N}} \subset s$ is an orthonormal sequence in $\ell_{2}$ and a Schauder basis of $\operatorname{im} V$. Therefore, $\Phi: \operatorname{im} V \rightarrow \lambda^{2}\left(\left|f_{j}\right|_{q}\right)$ defined by $\Phi f_{j}:=e_{j}$ for $j \in \mathbb{N}$ is a Fréchet space isomorphism (see [12, Lemma 27.25]), and so is $\Phi \circ V: \lambda^{2}(A) \rightarrow \lambda^{2}\left(\left|f_{j}\right|_{q}\right)$. But $(\Phi \circ V) e_{j}=e_{j}$ for $j \in \mathbb{N}$, hence $\lambda^{2}(A)=\lambda^{2}\left(\left|f_{j}\right|_{q}\right)$ as Fréchet ${ }^{*}$-algebras, whence $A \sim\left(\left|f_{j}\right|_{q}\right)_{j \in \mathbb{N}, q \in \mathbb{N}_{0}}$.

\section{Quasi-equivalence Conjecture}

In the last section we shall show how commutative subalgebras of $\mathcal{L}\left(s^{\prime}, s\right)$ are connected with the so-called Quasi-equivalence Conjecture stating that all bases in a nuclear Fréchet space are quasiequivalent (see [13, §8.3], [26] and references therein). Here we only consider the case (still unsolved) of closed subspaces of $s$, i.e. nuclear Fréchet spaces with the property (DN). Let us recall that two bases $\left(f_{j}\right)_{j \in \mathbb{N}}$ and $\left(g_{j}\right)_{j \in \mathbb{N}}$ of a Fréchet space $X$ are called quasi-eqivalent if there is a bijection $\sigma: \mathbb{N} \rightarrow \mathbb{N}$ and a sequence $\left(\lambda_{j}\right)_{j \in \mathbb{N}}$ of nonzero scalars such that the operator $T: X \rightarrow X$ defined by $T f_{j}=\lambda_{j} g_{\sigma(j)}$ is a Fréchet space isomorphism.

Theorem 15. Let $X$ be an infinite-dimensional closed subspace of s. Then the following assertions are equivalent. 
(i) For each two closed commutative ${ }^{*}$-subalgebras $E, F$ of $\mathcal{L}\left(s^{\prime}, s\right)$ we have: if $E \cong F \cong X$ as Fréchet spaces then $E \cong F$ as Fréchet ${ }^{*}$-algebras.

(ii) For each two basic sequences $\left(f_{j}\right)_{k \in \mathbb{N}}$ and $\left(g_{j}\right)_{k \in \mathbb{N}}$ of $s$ which are at the same time orthonormal sequences in $\ell_{2}$ we have: if $\lambda^{2}\left(\left|f_{j}\right|_{q}\right) \cong \lambda^{2}\left(\left|g_{j}\right|_{q}\right) \cong X$ as Fréchet spaces then there is a bijection $\sigma: \mathbb{N} \rightarrow \mathbb{N}$ such that $\left(\left|f_{j}\right|_{q}\right)_{j \in \mathbb{N}, q \in \mathbb{N}_{0}} \sim\left(\left|g_{\sigma(j)}\right|_{q}\right)_{j \in \mathbb{N}, q \in \mathbb{N}_{0}}$.

(iii) For each two Köthe matrices $A$ and $B$ such that $A \sim A^{2}$ and $B \sim B^{2}$ we have: if $\lambda^{2}(A) \cong$ $\lambda^{2}(B) \cong X$ as Fréchet spaces then there is a bijection $\sigma: \mathbb{N} \rightarrow \mathbb{N}$ such that $A \sim B_{\sigma}$.

(iv) In $X$ all bases are quasi-equivalent.

Proof. (i) $\Leftrightarrow\left(\right.$ ii): If $E$ and $F$ are closed commutative ${ }^{*}$-subalgebras of $\mathcal{L}\left(s^{\prime}, s\right)$ then, by Theorem 4. there are basic sequences $\left(f_{j}\right)_{j \in \mathbb{N}}$ and $\left(g_{j}\right)_{j \in \mathbb{N}}$ of $s$ which are at the same time orthonormal in $\ell_{2}$ such that $E \cong \lambda^{2}\left(\left|f_{j}\right|_{q}\right)$ and $F \cong \lambda^{2}\left(\left|g_{j}\right|_{q}\right)$ as Fréchet *algebras. Moreover, for each such a sequence $\left(f_{j}\right)_{j \in \mathbb{N}}, \lambda^{2}\left(\left|f_{j}\right|_{q}\right)$ is isomorphic as a Fréchet ${ }^{*}$-algebra to a closed commutative ${ }^{*}$-subalgebra of $\mathcal{L}\left(s^{\prime}, s\right)$. Therefore, assuming $\lambda^{2}\left(\left|f_{j}\right|_{q}\right) \cong \lambda^{2}\left(\left|g_{j}\right|_{q}\right) \cong X$ as Fréchet spaces, it follows from (i) and [4, Prop. 4.2] that $\left(\left|f_{j}\right|_{q}\right)_{j \in \mathbb{N}, q \in \mathbb{N}_{0}} \sim\left(\left|g_{\sigma(j)}\right|_{q}\right)_{j \in \mathbb{N}, q \in \mathbb{N}_{0}}$. Conversely, if we assume that $E \cong F \cong X$ as Fréchet spaces then, by (ii) and again by [4, Prop. 4.2], we get that $E \cong F$ as Fréchet ${ }^{*}$-algebras.

(i) $\Leftrightarrow($ iii): This is an immediate consequence of Theorem 4 and [4, Prop. 4.2].

(iii) $\Rightarrow$ (iv): Let $\left(x_{j}\right)_{j \in \mathbb{N}},\left(y_{j}\right)_{j \in \mathbb{N}}$ be two bases in $X$. Then

$$
\lambda^{2}(A) \cong \lambda^{2}(B) \cong X
$$

as Fréchet spaces, where

$$
A:=\left(\frac{\left|x_{j}\right|_{q}}{\left\|x_{j}\right\|_{\ell_{2}}}\right)_{j \in \mathbb{N}, q \in \mathbb{N}_{0}} \text { and } B:=\left(\frac{\left|y_{j}\right|_{q}}{\left\|y_{j}\right\|_{\ell_{2}}}\right)_{j \in \mathbb{N}, q \in \mathbb{N}_{0}} .
$$

Then for all $q \in \mathbb{N}_{0}$ we have

$$
\left(\frac{\left|x_{j}\right|_{q}}{\left\|x_{j}\right\|_{\ell_{2}}}\right)^{2} \leq \frac{\left\|x_{j}\right\|_{\ell_{2}}\left|x_{j}\right|_{2 q}}{\left\|x_{j}\right\|_{\ell_{2}}^{2}}=\frac{\left|x_{j}\right|_{2 q}}{\left\|x_{j}\right\|_{\ell_{2}}}
$$

so $A^{2} \prec A$. Moreover, since

$$
\frac{\left|x_{j}\right|_{q}}{\left\|x_{j}\right\|_{\ell_{2}}} \geq 1
$$

for all $q \in \mathbb{N}_{0}$, we have also $A \prec A^{2}$, and thus $A \sim A^{2}$. Similarly, we show that $B \sim B^{2}$. Therefore, by (iii), there is a bijection $\sigma: \mathbb{N} \rightarrow \mathbb{N}$ such that $A \sim B_{\sigma}$. This means that

$$
\forall q \in \mathbb{N}_{0} \exists r \in \mathbb{N}_{0} \exists C>0 \forall j \in \mathbb{N} \quad\left|x_{j}\right|_{q} \leq C \lambda_{j}\left|y_{\sigma(j)}\right|_{r}
$$

and

$$
\forall r^{\prime} \in \mathbb{N}_{0} \exists q^{\prime} \in \mathbb{N}_{0} \exists C^{\prime}>0 \forall j \in \mathbb{N} \quad \lambda_{j}\left|y_{\sigma(j)}\right|_{r^{\prime}} \leq C^{\prime}\left|x_{j}\right|_{q^{\prime}},
$$

where $\lambda_{j}:=\frac{\left\|x_{j}\right\|_{\ell_{2}}}{\left\|y_{\sigma(j)}\right\|_{\ell_{2}}}$. Hence $x_{j} \mapsto \lambda_{j} y_{\sigma(j)}, j \in \mathbb{N}$, defines an isomorphism of $X$, i.e. the bases $\left(x_{j}\right)_{j \in \mathbb{N}}$ and $\left(y_{j}\right)_{j \in \mathbb{N}}$ are quasi-equivalent.

(iv) $\Rightarrow$ (ii): Assume that in $X$ all bases are quasi-equivalent. Let $\left(f_{j}\right)_{j \in \mathbb{N}}$ and $\left(g_{j}\right)_{j \in \mathbb{N}}$ be basic sequences of $s$ which are at the same time orthonormal sequences of $\ell_{2}$ and such that $\lambda^{2}\left(\left|f_{j}\right|_{q}\right) \cong$ $\lambda^{2}\left(\left|g_{j}\right|_{q}\right) \cong X$ as Fréchet spaces. Let $F$ and $G$ be closed linear span in $s$ of $\left(f_{j}\right)_{j \in \mathbb{N}}$ and $\left(g_{j}\right)_{j \in \mathbb{N}}$, respectively. Then $F \cong G \cong X$ as Fréchet spaces; let $T: G \rightarrow F$ be an isomorphism. Clearly, $\left(F_{j}\right)_{j \in \mathbb{N}}$, $F_{j}:=T g_{j}$, is a basis in $F$ which is, by assumption quasi-equivalent to $\left(f_{j}\right)_{j \in \mathbb{N}}$. Consequently, there is a sequence $\left(\lambda_{j}\right)_{j \in \mathbb{N}}$ of nonzero scalars and a bijection $\sigma: \mathbb{N} \rightarrow \mathbb{N}$ such that $S: F \rightarrow F$ defined by sending $F_{\sigma(j)}$ to $\lambda_{j}^{-1} f_{j}$ is an isomorphism of Fréchet spaces.

Now, let $V:=S T: G \rightarrow F$ and define $\|\cdot\|_{V}: F \rightarrow[0, \infty)$, by $\|V \xi\|_{V}:=\|\xi\|_{\ell_{2}}$. Since $\|\cdot\| \ell_{\ell_{2}}$ is a dominating Hilbert norm on $G,\|\cdot\|_{V}$ is a dominating Hilbert norm on $F$ and, consequently, 
$\left(\lambda_{j}^{-1} f_{j}\right)_{j \in \mathbb{N}}=\left(V g_{\sigma(j)}\right)_{j \in \mathbb{N}}$ is an orthonormal sequence in $F$ with respect to the Hilbert norm $\|\cdot\|_{V}$. In particular, ||$f_{j} \|_{V}=\left|\lambda_{j}\right|$ for $j \in \mathbb{N}$ and thus, $\|\cdot\|_{V}$ being a dominating norm on $F$,

$$
\forall q \in \mathbb{N}_{0} \exists r \in \mathbb{N}_{0}, C>0 \forall j \quad\left|f_{j}\right|_{q} \leq\left. C|| f_{j}\right|_{V}\left|f_{j}\right|_{r}=C\left|\lambda_{j}\right|\left|f_{j}\right|_{r} .
$$

Moreover, since $\|\cdot\|_{V}$ is a continuous norm on $F$,

$$
\exists r_{0} \in \mathbb{N}_{0}, C_{0}>0 \forall j \quad\left|\lambda_{j}\right|=|| f_{j} \|_{V} \leq C_{0}\left|f_{j}\right|_{r_{0}} .
$$

Now, since

$$
\forall q \in \mathbb{N}_{0} \exists r \in \mathbb{N}_{0}, C>0 \forall j \in \mathbb{N} \quad\left|f_{j}\right|_{q}^{2} \leq C\left|f_{j}\right|_{r}
$$

it follows that

$$
\forall q \in \mathbb{N}_{0} \exists r \in \mathbb{N}_{0}, C>0 \forall j \quad\left|\lambda_{j}\right|\left|f_{j}\right|_{q} \leq C_{0}\left|f_{j}\right|_{r_{0}}\left|f_{j}\right|_{q} \leq C_{0}\left|f_{j}\right|_{\max \left\{r_{0}, q\right\}}^{2} \leq C\left|f_{j}\right|_{r} .
$$

Finally, let $W: F \rightarrow F$ be defined by $f_{j} \mapsto \lambda_{j} f_{j}$ for $j \in \mathbb{N}$. Then, by (13) and (14), $W$ is an automorphism of the Fréchet space $F$, and thus $W V$ is an isomorphism of Fréchet spaces which sends $g_{\sigma(j)}$ to $f_{j}$. This, clearly, implies that $\left(\left|f_{j}\right|_{q}\right)_{j \in \mathbb{N}, q \in \mathbb{N}_{0}} \sim\left(\left|g_{\sigma(j)}\right|_{q}\right)_{j \in \mathbb{N}, q \in \mathbb{N}_{0}}$.

For a monotonically increasing sequence $\alpha=\left(\alpha_{j}\right)_{j \in \mathbb{N}}$ in $[0, \infty)$ such that $\lim _{j \rightarrow \infty} \alpha_{j}=\infty$ we define the power series space of infinite type

$$
\Lambda_{\infty}(\alpha):=\left\{\left(\xi_{j}\right)_{j \in \mathbb{N}} \subset \mathbb{C}^{\mathbb{N}}: \sum_{j=1}^{\infty}\left|\xi_{j}\right|^{2} e^{2 q \alpha_{j}}<\infty \quad \text { for all } q \in \mathbb{N}_{0}\right\} .
$$

It appears that the space $\Lambda_{\infty}(\alpha)$ is nuclear if and only if $\sup _{j \in \mathbb{N}} \frac{\log j}{\alpha_{j}}<\infty$ (see [12, Prop. 29.6]). As a consequence, of Theorem [15, we get another proof of the following well-known result of Mityagin [13. Th. 12].

Corollary 16 (Mityagin 1961). In nuclear power series space of infinite type $\Lambda_{\infty}(\alpha)$ all bases are quasi-equivalent.

Proof. Let $E, F$ be a closed commutative ${ }^{*}$-subalgebras of $\mathcal{L}\left(s^{\prime}, s\right)$ isomorphic as Fréchet spaces to $\Lambda_{\infty}(\alpha)$. Then, by [4, Cor. 6.10], $E$ and $F$ are isomorphic to $\Lambda_{\infty}(\alpha)$ as a Fréchet ${ }^{*}$-algebra, and thus, by Theorem [15, all bases in $\Lambda_{\infty}(\alpha)$ are quasi-equivalent.

Since, by Corollary 8, every closed subspace of $s$ with basis is isomorphic as a Fréchet space to some closed commutative ${ }^{*}$-subalgebra of $\mathcal{L}\left(s^{\prime}, s\right)$, we can rewrite Theorem [15] in the following way.

Corollary 17. The following assertions are equivalent.

(i) For each two closed commutative ${ }^{*}$-subalgebras $E, F$ of $\mathcal{L}\left(s^{\prime}, s\right)$ we have: if $E \cong F$ as Fréchet spaces then $E \cong F$ as Fréchet*-algebras.

(ii) For each two basic sequences $\left(f_{j}\right)_{k \in \mathbb{N}}$ and $\left(g_{j}\right)_{k \in \mathbb{N}}$ of $s$ which are at the same time orthonormal sequences in $\ell_{2}$ we have: if $\lambda^{2}\left(\left|f_{j}\right|_{q}\right) \cong \lambda^{2}\left(\left|g_{j}\right|_{q}\right)$ as Fréchet spaces then there is a bijection $\sigma: \mathbb{N} \rightarrow \mathbb{N}$ such that $\left(\left|f_{j}\right|_{q}\right)_{j \in \mathbb{N}, q \in \mathbb{N}_{0}} \sim\left(\left|g_{\sigma(j)}\right|_{q}\right)_{j \in \mathbb{N}, q \in \mathbb{N}_{0}}$.

(iii) For each two Köthe matrices $A$ and $B$ such that $A \sim A^{2}$ and $B \sim B^{2}$ we have: if $\lambda^{2}(A) \cong$ $\lambda^{2}(B)$ as Fréchet spaces then there is a bijection $\sigma: \mathbb{N} \rightarrow \mathbb{N}$ such that $A \sim B_{\sigma}$.

(iv) In each closed subspace of s all bases are quasi-equivalent.

Acknowledgements. I cordially thank Leonhard Frerick for many valuable suggestions and encouraging me to solve the problems raised in the present paper. 


\section{REFERENCES}

[1] S. J. Bhatt, A. Inoue, H. Ogi, Spectral invariance, K-theory isomorphism and an application to the differential structure of $C^{*}$-algebras. J. Operator Theory 49 (2003), no. 2, 389-405.

[2] T. Ciaś, On the algebra of smooth operators. Studia Math. 218 (2013), no. 2, 145-166.

[3] T. Ciaś, Algebra of smooth operators. PhD dissertation, A. Mickiewicz University, Poznań, 2014. Available at https://repozytorium.amu.edu.pl/jspui/bitstream/10593/10958/1/phd_thesis_TCias.pdf

[4] T. Ciaś, Commutative subalgebras of the algebra of smooth operators. Monatsh. Math. 179 (2016), no. 4, 1-23.

[5] L. Crone, W.B. Robinson, Every nuclear Fréchet space with a regular basis has the quasi-equivalence property. Studia Math. 52 (1974/75), 203-207.

[6] J. Cuntz, Bivariante K-Theorie für lokalkonvexe Algebren und der Chern-Connes-Charakter. Doc. Math. 2 (1997), 139-182.

[7] J. Cuntz, Cyclic theory and the bivariant Chern-Connes character. Noncommutative geometry, Lecture Notes in Math., 1831, Springer, Berlin, 2004, 73-135.

[8] P. Domański, Algebra of smooth operators. Unpublished note available at www.staff.amu.edu.pl/ domanski/salgebra1.pdf

[9] G. A. Elliot, T. Natsume, R. Nest, Cyclic cohomology for one-parameter smooth crossed products. Acta Math. 160 (1998), 285-305.

[10] H. Glöckner, B. Langkamp, Topological algebras of rapidly decreasing matrices and generalizations. Topology Appl. 159 (2012), no. 9, 2420-2422.

[11] A.Yu. Helemskii, The Homology of Banach and Topological Algebras. Mathematics and its Applications (Soviet Series), 41. Kluver Academic Publishers, Dordrecht, 1989.

[12] R. Meise, D. Vogt, Introduction to functional analysis. Oxford University Press, New York 1997.

[13] B.S. Mityagin, Approximate dimension and bases in nuclear spaces. (Russian) Uspehi Mat. Nauk 16 (1961), no. 4 (100), 63-132.

[14] N.C. Phillips, K-theory for Fréchet algebras. Internat. J. Math. 2 (1991), no. 1, 77-129.

[15] A. Yu. Pirkovskii, Homological dimensions and approximate contractibility for Köthe algebras. Banach algebras 2009, 261-278, Banach Center Publ., 91, Polish Acad. Sci. Inst. Math., Warsaw, 2010.

[16] K. Piszczek, One-sided ideals of the non-commutative Schwartz space. Monatsh. Math. 178 (2015), no.4, 599-610.

[17] K. Piszczek, A Jordan-like decomposition in the noncommutative Schwartz space. Bull. Aust. Math. Soc. 91 (2015), no. 2, 322-330.

[18] K. Piszczek, Automatic continuity and amenability in the non-commutative Schwartz space. J. Math. Anal. Appl. 432 (2015), no. 2, 954-964.

[19] K. Piszczek, Corrigendum to "Automatic continuity and amenability in the non-commutative Schwartz space" [J. Math. Anal. Appl. 432 (2015), no. 2, 954-964]. J. Math. Anal. Appl. 435 (2016), no. 1, 1015-1016.

[20] H. H. Schaefer, Topological vector spaces. Third printing corrected. Graduate Texts in Mathematics, Vol. 3. SpringerVerlag, New York-Berlin, 1971.

[21] D. Vogt, Charakterisierung der Unterräume von s. Math. Z. 155 (1977), 109-117.

[22] D. Vogt, Subspaces and quotient spaces of $(s)$. Functional analysis: surveys and recent results (Proc. Conf., Paderborn, 1976), North-Holland Math. Studies, Vol. 27; Notas de Mat., No. 63, North-Holland, Amsterdam, 1977, $167-187$.

[23] D. Vogt Eine Charakterisierung der Potenzreihenräume von endlichem Typ und ihre Folgerungen. Manuscripta Math. 37 (1982), no. 3, 269-301.

[24] D. Vogt, On the functors $\operatorname{Ext}^{1}(E, F)$ for Fréchet spaces. Studia Math. 85 (1987), no. 2, $163-197$.

[25] D. Vogt, Unitary endomorphims of power series spaces. Math. Forum, Vol. 7, Studies on Mathematical Analysis, Vladikavkaz, (2013), 220-239.

[26] N. Zobin, Some remarks on quasi-equivalence of bases in Fréchet spaces. Linear Algebra Appl. 307 (2000), no. 1-3, $47-67$.

T. Ciaś

Faculty of Mathematics and Computer Science

Adam Mickiewicz University in Poznań

Umultowska 87

61-614 Poznań, POLAND

e-mail: tcias@amu.edu.pl 